\title{
Aspectos epidemiológicos do Lynxacarus radovskyi - Revisão de literatura
}

\author{
Epidemiological aspects of Lynxacarus radovskyi - Literature review \\ Aspectos epidemiológicos de Lynxacarus radovskyi - Revisión de la literatura
}

Caio Cezar Nogueira de Souza ORCID: https://orcid.org/0000-0003-3149-6662 Universidade Federal Rural da Amazônia, Brasil E-mail: caionnogueira@gmail.com Edigleise Costa Figueiredo ORCID: https://orcid.org/0000-0002-9123-3373 Universidade Federal Rural da Amazônia, Brasil E-mail: edi.vete20@gmail.com

Rafaela Sena Vasconcelos ORCID: https://orcid.org/0000-0002-1613-5650 Universidade Federal Rural da Amazônia, Brasil E-mail:rafaelasv.vet@gmail.com

Andre Marcelo Conceição Meneses ORCID: https://orcid.org/0000-0003-3494-2998 Universidade Federal Rural da Amazônia, Brasil E-mail: andre.meneses1974@icloud.com

Sinerey Karla Salim Aragão de Sousa ORCID: https://orcid.org/0000-0002-3994-9358 Universidade Federal Rural da Amazônia, Brasil E-mail: karlasalim6@gmail.com

\begin{abstract}
Resumo
Enfermidades dermatológicas vêm ganhando cada vez mais destaque na rotina de clínicas e hospitais veterinários, e dentro desse contexto a linxacariose causada pelo ácaro Lynxacarus radovskyi tem apresentado uma ocorrência significativa em felinos domésticos. Dessa forma, o objetivo desse estudo é fazer uma revisão de literatura narrativa acerca desse agente, abordando a epidemiologia, métodos diagnósticos e tratamento. Por fim, considera-se que o intuito principal do estudo foi alcançado, tratando-se de um agente que não é comumente encontrado em animais domésticos, contudo é de extrema importância o conhecimento acerca de suas causas, epidemiologia, protocolos terapêuticos e profilaxia a fim de controlar sua prevalência e as taxas de infecção em seus hospedeiros.
\end{abstract}

Palavras-chave: Ácaro; Dermatologia; Felinos domésticos.

\begin{abstract}
Dermatological diseases are increasingly gaining prominence in the routine of veterinary clinics and hospitals, and within this context, the lynxacariasis caused by the mite Lynxacarus radovskyi has presented a significant occurrence in domestic cats. Thus, the aim of this study is to make a narrative literature review about this agent, addressing the epidemiology, diagnostic methods and treatment. Finally, it is considered that the main purpose of the study was achieved, since it is an agent that is not commonly found in domestic animals; however, it is extremely important to know about its causes, epidemiology, therapeutic protocols and prophylaxis in order to control its prevalence and infection rates in its hosts.
\end{abstract}

Keywords: Mite; Dermatology; Domestic feline.

\section{Resumen}

Las enfermedades dermatológicas están ganando cada vez más protagonismos en la rutina de las clínicas y hospitales veterinarios, y dentro de este contexto, la linxacariasis causada por el ácaro Lynxacarus radovskyi ha presentado una importante incidencia en los gatos domésticos. De esta forma, el objetivo de este estudio es hacer una revisión de la literatura narrativa sobre este agente, abordando la epidemiología, los métodos de diagnóstico y el tratamiento. Finalmente, se considera que el objetivo principal del estudio se logró, ya que es un agente que no se encuentra comúnmente en los animales domésticos; sin embargo, es sumamente importante conocer sus causas, epidemiología, protocolos terapéuticos y profilaxis para controlar su prevalencia y las tasas de infección en sus huéspedes.

Palabras clave: Ácaro; Dermatología; Felino doméstico. 


\section{Introdução}

Dentre as inúmeras doenças que acometem os animais domésticos, destacam-se as enfermidades dermatológicas causadas por variados agentes e que correspondem a uma grande parte de atendimentos de clínicas e hospitais veterinários, se apresentando como infecções que possuem etiologias diversas que variam desde adquiridas até as de origem parasitárias, fúngicas ou bacterianas (Bellato et al., 2003; Paula, 2019; Santos et al., 2020).

Dentro desse contexto, a linxacariose surge como uma doença que primariamente era incomum em felinos, mas que com o passar dos anos tem se tornado frequente em atendimentos destinados a essa espécie, se manifestando de forma sintomática ou assintomática, reforçando a necessidade de diagnósticos e investigações mais precisas em torno do agente causador chamado de Lynxacarus radovskyi (Faustino et al., 2004; Oliveira \& Vieira Neto, 2019).

Partindo desse pressuposto, diante da ocorrência do ácaro em diversas regiões do Brasil e do mundo, julgam-se que fatores como clima, precisão no diagnóstico, terapia medicamentosa estabelecida e medidas profiláticas são variáveis que tem influência direta na incidência desse ectoparasita (Oliveira \& Vieira Neto, 2019; Lopes et al., 2021).

Dessa forma, objetiva-se atualizar as informações acerca da linxacariose e sobre seu agente etiológico L. radovsky no que tange a distribuição geográfica, epidemiologia, patogenia e resposta imunológica, diagnóstico, sinais clínicos e abordagem terapêutica associada a profilaxia.

\section{Metodologia}

Trata-se de uma revisão de literatura narrativa, que de acordo com Sousa et al. (2018), possui as seguintes etapas: seleção do tema de revisão, pesquisa na literatura, leitura e análise, redação da revisão e referencias, os artigos podem abranger vários estudos, além de apresentar uma descrição muito ampla, sendo assim menos abrangentes. Além disso, mesmo sendo considerado de menor impacto científico devido a escolha arbitraria de artigos e por estar sujeita a viés de seleção, esse tipo de método de pesquisa é considerado essencial no debate de determinadas temáticas, contribuindo com novas questões e proporcionando a atualização do conhecimento (Rother, 2007; Cordeiro et al., 2007).

Esta pesquisa utilizou a base de dados Google acadêmico e Scielo (Scientific Eletronic Libary Online), sem restrição de período. Dessa forma, foram encontrados estudos que abordavam desde a descoberta do ácaro, bem como sua propagação e incidência em diversas regiões do mundo, além dos efeitos clínicos da infecção na espécie felina associado a diferentes tratamentos realizados.

O estudo foi realizado nos meses de novembro de 2021 a janeiro de 2022, utilizando como forma de levantamento as seguintes palavras-chave: Lynxacarus radovskyi, dermatopatia em felinos, ácaros em animais domésticos. Como critério de inclusão foram escolhidos aqueles que as referências abordavam assuntos relacionados ao tema como: Artigos de revisão, livros, trabalhos de conclusão de curso, artigos originais e anais de congresso.

Todas as pesquisas foram resumidas e estão presentes na revisão de literatura com o intuito de alcançar os objetivos pretendidos. Trabalhos que fugiram do tema proposto, não obtinham resumo detalhado, abordavam outra espécie de ácaro ou não citavam a ocorrência do agente na espécie felina foram excluídos.

\section{Revisão de Literatura}

\subsection{Etiologia}

L. radovskyi é um ácaro pertencente à família Listrophoridae, que possui como sua característica anatômica um tamanho que varia em torno de $0,5 \mathrm{~mm}$ e entre 430 e $515 \mu \mathrm{c}$ de comprimento, propriedades essas que possibilitam a sua 
permanência na porção distal da haste pilosa do hospedeiro, fato que permite sua visualização a olho nu (Scoot et al., 1996; Faustino et al., 2004).

Além disso, Taman e Omar (2009) identificaram em sua pesquisa as características morfológicas desse agente, no qual constatou-se que as fêmeas adultas (figura 1) são maiores que os machos, e que o ácaro macho possui um quarto par de pernas muito aumentado, extremidade posterior alongada com dois processos que transportam um longo cabelo lateral (figura 2)

Figura 1: Lynxacarus radovskyi fêmea.

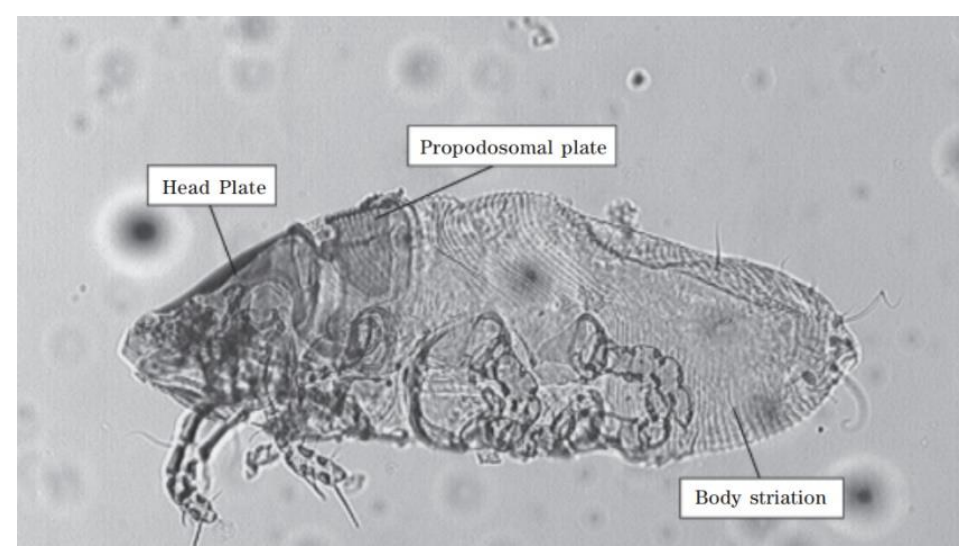

Fonte: Jeffery et al. (2012).

Figua 2: Lynxacarus radovskyi macho.

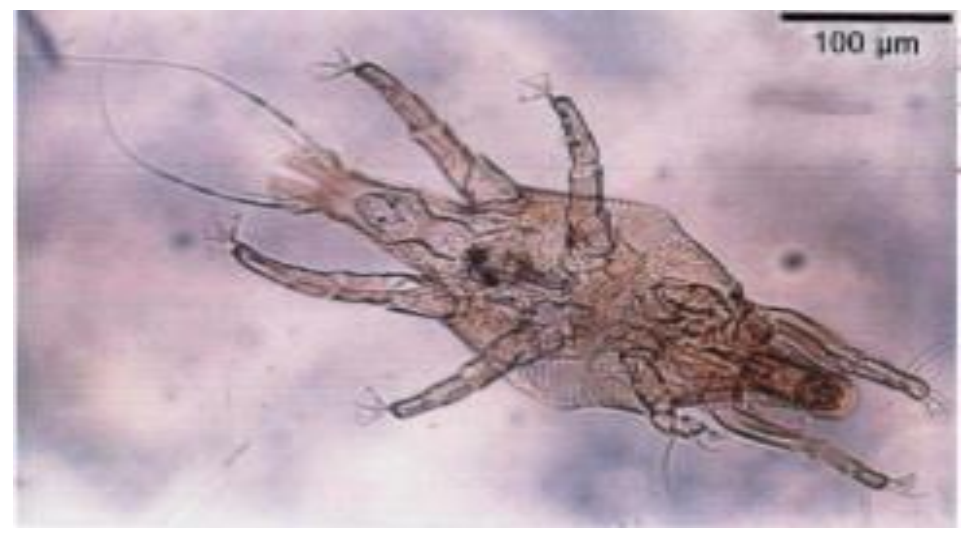

Fonte: Taman e Omar (2009).

\subsection{Distribuição geográfica}

No que diz respeito a presença geográfica do ácaro, o primeiro relato foi descrito por Tenório em 1974. Após isso, houve outros relatos em diferentes regiões do mundo, como na Austrália (Bowman \& Domrow, 1978), Flórida (Greve \& Gerrish, 1981), Brasil (Faccini \& Coutinho, 1986), Texas (Craig et al., 1993) e Porto Rico (Fox, 1997). Segundo Foley (1991), o parasita evolui melhor em condições climáticas subtropicais, contudo Craig et al. (1993), relata que o comportamento natural desse ácaro é mais bem evidenciado em regiões tropicais, não deixando totalmente claro quais ambientes predispõe a sua incidência

No Brasil, após o primeiro relato descrito por Faccini e Coutinho (1986) surgiram outros casos em Pernambuco, São Paulo, Pará, Rio de Janeiro e Paraíba (Pereira, 1996; Figueiredo et al., 2004; Jaffé et al., 2005; Ferreira et al., 2010). 
Além disso, Da Rocha et al. (2008), em um estudo sobre a ocorrência de ácaros em pequenos animais atendidos em um Hospital Veterinário localizado no Rio Grande do Norte, identificou L. radovsky em 4 felinos, correspondendo a 15,3\% do total de animais diagnosticados com algum tipo de doença dermatológica.

Outrossim, Lopes et al. (2021), identificou a presença do ectoparasita em 26 gatos oriundos de um gatil na cidade de Porto Velho, Rondônia. Dessa forma, observa-se que após o primeiro relato em território brasileiro os números de infecções causadas por esse agente foram sendo descritas de forma mais frequente na literatura, evidenciando sua presença em diversas regiões com condições climáticas e epidemiológicas variadas.

\subsection{Epidemiologia}

A forma de transmissão ocorre principalmente através do contato direto. Contudo, os fômites também são citados como uma importante via de transmissão entre esses animais (Foley, 1991; Craig et al., 1993; Scoot et al., 2001). Contudo, é de suma importância destacar que mesmo em uma população grande de felinos em determinado ambiente, apenas uma quantidade menor pode se infectar, haja vista que o agente não possui um alto poder de contágio (Faustino et al., 2004). Além disso, em alguns relatos de casos, há evidências da infecção em humanos que tiveram contato com gatos parasitados, ocasionando desta forma lesões dermatológicas (Foley, 1991).

Segundo Romeiro et al. (2007), há evidências que o agente possui condições de desenvolvimento fora do hospedeiro, observando que os estágios evolutivos proporcionam chances altas de sobrevivência. Em relação a faixa etária, segundo o estudo de Figueiredo et al. (2004) e Romeiro et al. (2007), o parasitismo desse ácaro teve maior ocorrência em felinos machos com idade variando de 1 ano a 7 anos.

No que diz respeito ao sexo, Figueiredo et al. (2004) observou em seu estudo maior incidência do parasitismo em machos, tal achado pode ser justificado pelo fato de felinos machos possuírem hábito maior de sair para rua, devido seu próprio instinto de caça, enquanto a fêmea é mantida mais restrita ao ambiente domiciliar com o intuito de evitar uma possível gestação.

Já Romeiro et al. (2007), aponta que não há ligação direta entre a ocorrência da doença e o sexo, pontuando que o contato com o ácaro pode sofrer influência de outras variáveis como a aglomeração, que pode facilitar o contato de animais sadios com infectados, aumentando as chances de contaminação.

\subsection{Diagnóstico}

A forma do diagnóstico do L. radosvky pode ser feita a olho nu de acordo Scoot et al. (2001) ou por avulsão do pelo da área mais afetada com auxílio da lupa para melhor visualização (Sloss et al., 1999).

Em um trabalho descrito por Silva et al. (2009), após esse primeiro passo o material coletado foi acondicionado em um envelope lacrado e encaminhado para o laboratório. Essa amostra foi colocada em uma lâmina com três gotas de hidróxido de potássio $10 \%$, e após o período de 10 minutos colocou-se uma lamínula sobre o material e posteriormente realizada a visualização em microscópio na objetiva de 10x, no qual fez-se a classificação do ácaro de acordo com as características morfológicas descritas por Faccini e Coutinho (1986). Diante desse método foi possível identificar o agente e estabelecer a terapêutica adequada para o animal (Silva et al., 2009).

Desse modo, pode-se dizer que existem variados métodos diagnósticos para a confirmação do quadro clínico dessa enfermidade, dentre eles o exame microscópico das escamas, com o auxílio de fita adesiva, lâmpada de Wood e tricrograma. Além disso, é importante que se descarte outras enfermidades cutâneas como otocaríase, pediculose, queiletielose, sarna notoédrica (Larsson \& Lucas, 2016). 
Dessa forma, preconiza-se que o diagnóstico definitivo pode ser feito com o isolamento do agente patogênico (Pereira, 1996; Wilkinson \& Harvey, 1996) ou até mesmo com a utilização de outros métodos diagnósticos com boa eficácia como o raspado cutâneo ou impressão com fita de acetato (Scott et al., 2001; Medleau \& Hnilica, 2003).

\subsection{Sinais clínicos}

Embora a maior parte das infecções pelo ácaro L. radovsky se manifeste de forma subclínica, quando visíveis, os sinais clínicos apresentados variam no que diz respeito a intensidade, de acordo com o parasitismo do ácaro no animal examinado e do tempo de duração da infestação (Wilkison \& Harvey, 1996; Pereira, 1996; Scoot et al., 2001).

Os sintomas clínicos mais relatados da infecção por esse agente são pruridos com intensidade variada, pelagem que lembra o aspecto de "sal e pimenta" no pelo do animal, além de possíveis áreas alopécicas que podem ser justificadas pela facilidade da queda de pelo no manuseio do animal infectado, podendo surgir caspas e dermatite miliar (Greve \& Gerrish, 1981; Payne et al., 2005).

Ademais, segundo Foley (1991) o animal pode manifestar distúrbios gastrointestinais em decorrência do excesso de lambedura, podendo ocasionar êmese, irritação ou prolapsos retais, constipação e bolas de pelo.

\subsection{Tratamento}

A literatura aponta diversos tipos de tratamento que variam tanto quanto ao uso de diferentes fármacos com protocolos terapêuticos variáveis, bem como diferentes vias de administração que demonstram eficácia no combate ao agente e na atenuação de manifestações clínicas (Oliveira \& Vieira Neto, 2019).

Diante das inúmeras alternativas presentes no mercado pet para o tratamento de infecções dermatológicas causadas por ectoparasitas, destaca- se nesse caso o uso de Selamectina, Imidacloprida, Fipronil e xampu a base de piretrina e carbaryl pó a 5\%, tais medicamento apresentam certa eficácia quando utilizados na terapêutica destinada para o combate ao $L$. radovskyi, sendo o Fipronil e a Selemectina os principais fármacos usados no tratamento da doença causada por esse agente, haja vista a melhora evidente do quadro clínico do animal infectado e a margem de segurança oferecida por esses princípio ativo (Faustino et al., 2004; Silva et al., 2009).

Outras opções presentes no âmbito veterinário consistem na utilização de um protocolo com associação de Moxidectina imidacloprida e fluralaner oral, tratamento este testado em um estudo conduzido por Han et al. (2016) na Malásia, no qual demonstrou eficácia quando destinado ao tratamento contra esse ectoparasita em felinos domésticos.

Além do uso oral, há também terapêuticas realizadas com fluralaner absorvido por via transdérmica, fornecendo eficácia inseticida e acaricida em felinos (Rohdich et al., 2014; Gassel et al., 2014) podendo ser uma boa alternativa medicamentosa para o tratamento de linxacariose.

Ademais, quando o protocolo está associado com banhos utilizando Amitraz o Fipronil teve sua eficácia potencializada. Segundo Lopes et al. (2021), após a utilização desse protocolo terapêutico, os animais em questão apresentaram, depois de um período de um mês, completa regressão da infecção e do quadro clínico.

Um estudo realizado por Campos et al. (2020), constatou que a utilização do medicamento Sarolaner em uma única dose por via oral demonstrou uma boa porcentagem de eficácia, em torno de $95 \%$, se apresentando como mais uma boa alternativa para o tratamento de felinos infectados por esse ácaro.

\section{Conclusão}

Dessa forma, pode-se concluir que a linxacariose tornou-se uma enfermidade mais frequente na medicina felina desde seu primeiro relato descrito no Brasil, sendo alvo de mais estudos acerca da ocorrência, diagnósticos e tratamentos. Diante 
disso, faz-se necessário a investigação acerca da forma de reprodução do ácaro, assim como os fatores que favorecem isso, a fim de proporcionar aos animais a utilização de terapias medicamentosas cada vez mais eficazes, bem como métodos diagnósticos precisos para identificar o ácaro independente da sintomatologia clínica do hospedeiro e da vida do agente.

Por fim, é importante a realização de trabalhos futuros acerca da patogenia do agente no hospedeiro, a fim de incentivar a descoberta de novos protocolos terapêuticos e medidas de prevenção, diminuindo assim a ocorrência da enfermidade causada por esse ectoparasita na espécie felina.

\section{Referências}

Bellato, V., Sartor, A. A., Souza, A. P. \& Ramos, B. C. (2003). Ectoparasitos em caninos do município de Lages, Santa Catarina, Brasil. Revista Brasileira de Parasitologia Veterinária, 12 (3), 95-98.

Bowman, W.L. \& Domrow R. (1978). The cat fur-mite (Lynxacarus radovskYI) in Australia. Australian Veterinary Journal, 54 (8), $403-404$.

Campos, D. R., Chaves, J. K. O., Assis, R. C. P., Fernandes, J. L. \& Scott, F. B. (2020). Efficacy of oral sarolaner against Lynxacarus radovskyi in naturally infested cats. Veterinary Dermatology, 31 (5), 355-e92. https://doi.org/10.1111/vde.12857

Cordeiro, A. M., Oliveira, G. M., Rentería, J. M. \& Guimarães, S. A. (2007). Revisão sistemática: uma revisão narrativa. Revista do Colégio Brasileiro de Cirurgiões, 34(6) 428-431. https://doi.org/10.1590/S0100-69912007000600012

Craig, T.M., Tell, P.D., Dubuisson, L. M. \& Dubuisson, R. K. (1993). Lynxacarus radovskyi infestation in a cat. Journal of American Veterinary Medical Association, 202 (4), 613-614.

Da Rocha, G. S., Ahid, S. M. M., Bezerra, A. C. D. S., Filgueira, K. D. \& Santos, J. P. S. (2008) Frequência de ácaros em cães e gatos no município de Mossoró, Rio Grande do Norte. Acta Scientiae Veterinariae, 36 (3), 263-266.

Facinni, J. L. H. \& Coutinho, V. (1986). Ocorrência de Lynxacarus radovskyi (Acari: Listrophoridae) em gatos domésticos no Brasil. Arquivos da Universidade Federal Rural do Rio de Janeiro, 9(1), 91- 93.

Faustino, M. A. G., Messias, J. B. \& Romeiro, E. T. (2004). Infestação por Lynxacarus radovskyi(Tenório, 1974) em felinos - revisão. Clínica Veterinária, 53(1),52-56.

Ferreira, D. R. A., Alves, L. C. \& Faustino, M. A. G., (2010). Ectoparasitos de Felis catus domesticus na cidade de João Pessoa, Paraiba, Brasil. Biotemas, 23(4), 43-50. https://doi.org/10.5007/2175-7925.2010v23n4p43

Figueiredo, F. B., Schubach, T. M. P., Pereira, S. A., Okamoto, T., Santos, I. B., Leme, L. R. P. \& Faccini, J. L. H. (2004). Nota De Pesquisa Relato De Mais 42 Casos De Linxacariose Felina Na Região Metropolitana Da Cidade Do Rio De Janeiro, RJ, Brasil. Revista Brasileira de Parasitologia Veterinária,13 (1), $41-43$.

Foley, R. H. (1991). An epizootic of rare fur mite in an island's cat population. Feline Practice, 19(3),17-19.

Fox, I. (1977). Felistrophorus, a new genus of mites on cats in Puerto Rico (Acarina: Listrophoridae). Proceedings of the Entomological Society of Washington, 79(1), 242-244.

Gassel M., Wolf, C., Noack, S., Williams, H \& LLG, T. (2014). The novel isoxazoline ectoparasiticide fluralaner: selective inhibition of arthropod Yaminobutyric acid- and L-glutamate-gated chloride channels and insecticidal/acaricidal activity. Insect Biochemistry and Molecular Biology, 45(1), 111-124. https://doi.org/10.1016/j.ibmb.2013.11.009

Greve, J. H. \& Gerrish, R. R. (1981). Fur mites (Lynxacarus ) from cats in Florida. Feline Practice, 11(6), 28-30.

Han, H. S., Noli, C. \& Cena, T. (2016). Efficacy and duration of action of oral fluralaner and spot-on moxidectin/imidacloprid in cats infested with Lynxacarus radovskyi. Veterinary dermatology, 27(6), 474-e127. https://doi.org/10.1111/vde.12390

Jaffé, E., Grillo, S., Costa, C. L. A., Vaz, C. E. S., Alves, L. C \& Almosny, N. R. P. (2005). Infestação de Lynxacarus radoviskyi em cães e gatos domésticos na cidade de Niterói (RJ): Relato de caso. Revista Brasileira de Ciência Veterinária, 12(3),110-113. http://dx.doi.org/10.4322/rbcv.2014.315

Jeffery, J., Norhidayu, S., Mohd Zain, S. N., Noor Hayati, M. I. \& Nurazila, B. (2012). Nota de pesquisa O ácaro de pele de gato, Lynxacarus radovskyi Tenorio, 1974 (Acarina: Astigmata: Listrophoridae) do gato, Felis catus na Malásia peninsular. Biomedicina tropical, 29(2), 308-310.

Larsson, C. E. \& Lucas, R. (2016). Tratado de medicina externa dermatologia veterinária. Interbook.

Lopes, T. V., De Souza, J. G. S. G., Pessôa, C. M., Leão, P. G. L., Schons, S. V. \& Sousa, F. A. (2021). Infestação por Lynxacarus radovskyi (Tenório, 1974) em gatos (Felis domestica), no Município de Porto Velho-RO. Research, Society and Development, 10(9), e35810918083-e35810918083. https://doi.org/10.33448/rsd-v10i9.18083

Medleau, L. \& Hnilica, K. A. (2003). Ácaro de pêlos de gatos (lynxacarus radovskyi). In: Medleau, L., Hnilica, K. A. Dermatologia de pequenos animais: atlas colorido e guia terapêutico. São paulo: Roca.

Oliveira, E. S. \& Vieira Neto, M. F. (2019). Linxacarose Felina: Revisão de Literatura, In: VI Encontro de Iniciação à Pesquisa, UNIFAMETRO 2019. Clínica e Biotecnologias aplicadas em Medicina Veterinária. 
Research, Society and Development, v. 11, n. 2, e48911226074, 2022

(CC BY 4.0) | ISSN 2525-3409 | DOI: http://dx.doi.org/10.33448/rsd-v11i2.26074

Paula, M. A. L. (2019). Levantamento epidemiológico das dermatopatias de cães e gatos atendidos no hospital veterinário da Universidade Federal de Uberlândia. Trabalho de Conclusão de Curso. Universidade Federal de Uberlândia. Uberlândia.

Payne, P. A., Dyden, M. W. \& Carter, G. R. (2005). External parasitic diseases of dogs and cats. A concise guide to infectious and parasitic diseases of dogs and cats. United States: Serviço Internacional de Informação Veterinária.

Pereira, M. C. P. (1996). The cat fur mite( Lynxacarus radovskyi ) in Brazil. Feline Practice, 24(5),24-26.

Rohdich N., Roepke R. K. A. \& Zschiesche E. (2014). A randomized, blinded, controlled and multi-centered field study comparing the efficacy and safety of Bravecto $^{\mathrm{TM}}$ (fluralaner) against Frontline ${ }^{\mathrm{TM}}$ (fipronil) in flea- and tick-infested dogs. Parasit Vectors, 7(83), 1-5. https://doi.org/10.1186/1756-3305-7-83

Romeiro, E. T., Alves, L. C., Soares, Y. M. V., Matoso, U. N. V. \& Faustino, M. A. G. (2007). Infestação por Lynxacarus radovskyi (Tenorio, 1974) em gatos domésticos procedentes da região metropolitana do Recife, Pernambuco, Brasil. Revista Brasileira de Parasitologia Veterinária, 16(3),159-162. https://doi.org/10.1590/S1984-29612007000300009

Rother, E. T. (2007). Revisão sistemática X revisão narrativa. Acta Paul Enfermagem, 20(1), 5-6.

Santos, A. J., Vieira, M. C. G., Lima, P. P. A., Oliveira, L. R. C., Cardinot, C. B., Rocha, T. V. P., \& Franciscato, C. (2020) Prevalência de microrganismos e ácaros encontrados em amostras dermatológicas e otológicas de cães e gatos. Revista Brasileira de Higiene e Sanidade Animal, 14(3),1-11.

Scott, D.W., Miller, H. W. \& Griffin, C. E. (1996). Doenças parasitárias da pele. In: Muller and Kirk Dermatologia dos pequenos animais. Manole. Scott, D. W., Craig, E. G. \& Miller, H. W. (2001). Kirk's small animal dermatology. W.B.Saunders Company.

Silva, M. F. O., Drechsler, L. E. M. L., Barros, A. M. M., Neves, A. K. R., Silva, F. M. F., Silva, E. N., Ramos, R. A. N., Faustino, M. A. G. \& Alves, L. C. (2009). Uso da selamectina para tratamento de dermatite felina por Lynxacarus radovskyi: relato de caso. Eventos UFRPE.

Sloss, M.W.; Kemp, R.L \& Zajac, A.M. (1999). Parasitologia clínica veterinária. Manole.

Sousa, L. M. M., Firmino, C. F., Marques-Vieira, C.M. A., Severino, S. S. P \& Pestana, H. C. F. C. (2018). Revisões da literatura científica: tipos, métodos e aplicações em enfermagem. Revista Portuguesa de Enfermagem de Reabilitação, 1(1),45-54. https://doi.org/10.33194/rper.2018.v1.n1.07.4391

Tamam, O. A. \& Omar, H.M (2009). Um novo recorde para o Lynxacarus na Toupeira Cega Menor egípcia (Spalax leucodon Egyptiacus). European Journal of Scientific Research, 33(3), 509-514.

Wilkinson, G. T. \& Harvey, R. G. (1996). Doenças Parasitárias. In:.Wilkinson, G. T., Harvey, R. G. Atlas colorido de dermatologia dos pequenos animais: guia para o diagnóstico.São Paulo: Manole. 